\title{
Intrapleural administration of gelatin-embedded, sustained-release basic fibroblast growth factor for the regeneration of emphysematous lungs in rats
}

\author{
Mitsumasa Kawago, MD, ${ }^{\text {a }}$ Tatsuya Yoshimasu, MD, PhD, ${ }^{a}$ Yasuhiko Tabata, PhD, DMedSci, DPharm, ${ }^{\text {b }}$ \\ Masaya Yamamoto, PhD, ${ }^{\mathrm{b}}$ Yoshimitsu Hirai, MD, PhD, ${ }^{\mathrm{a}}$ Takahiro Kinoshita, $\mathrm{MD}, \mathrm{PhD},{ }^{\mathrm{c}}$ and \\ Yoshitaka Okamura, $\mathrm{MD}, \mathrm{PhD}^{\mathrm{a}}$
}

\begin{abstract}
Background: Intra-airway and intra-arterial administration of gelatin-embedded, sustained-release basic fibroblast growth factor has stimulated regeneration of emphysematous lungs in animal experiments, but these routes of administration may also cause harm. This study investigated the effectiveness of intrapleural administration of gelatin-embedded, sustained-release basic fibroblast growth factor. This animal experiment preceded our clinical trial of intrapleural administration of sustained-release basic fibroblast growth factor in patients with chronic obstructive pulmonary disease accompanied by pneumothorax.
\end{abstract}

\begin{abstract}
Methods: Pulmonary emphysema was induced in Sprague-Dawley rats using porcine elastase. Gelatinembedded, sustained-release basic fibroblast growth factor was administered via the left pleural cavity. The rats were divided into a group that received gelatin-embedded, sustained-release basic fibroblast growth factor $\left(\mathrm{FGF}^{+}\right.$group, $\left.\mathrm{n}=6\right)$, and a group that did not $\left(\mathrm{FGF}^{-}\right.$group, $\left.\mathrm{n}=6\right)$. Animals were sacrificed after 14 days, and the results were evaluated by histologic examination.
\end{abstract}

Results: In the $\mathrm{FGF}^{+}$group, the mean linear intercept value of the alveolar septa was significantly shorter on the treated side than on the untreated side $(65.1 \pm 7.0 \mathrm{vs} 114.4 \pm 7.5 \mu \mathrm{m} ; P=.0005)$. There was no significant difference in the mean linear intercept value between the treated and untreated sides in the $\mathrm{FGF}^{-}$group.

Conclusions: Intrapleural administration of sustained-release basic fibroblast growth factor induced lung regeneration in rats with elastase-induced pulmonary emphysema. (J Thorac Cardiovasc Surg 2014;147:1644-9)

Pulmonary emphysema is characterized by permanent abnormal enlargement of the air spaces distal to the terminal bronchioles, with destruction of the terminal bronchiolar walls but no obvious fibrosis. ${ }^{1}$ Although medications and home oxygen therapy can help to control symptoms, there is no treatment available to reverse the changes in pulmonary structure, and the prognosis of patients with emphysema is, therefore, extremely poor.

Surgical treatments may improve pulmonary function, but they have disadvantages. Lung transplantation is the most effective treatment, but it is difficult because of a shortage of donors, the need for immunosuppression, and the development of bronchiolitis obliterans. ${ }^{2}$ Lung volume

\footnotetext{
From the Department of Thoracic and Cardiovascular Surgery, ${ }^{a}$ Wakayama Medical University, Wakayama, Japan; the Institute for Frontier Medical Sciences, ${ }^{b}$ Kyoto University, Kyoto, Japan; and the Department of Thoracic and Cardiovascular Surgery, ${ }^{\mathrm{c}}$ Minami Wakayama Medical Center, Tanabe, Japan.

This study was supported by a Grant-in-Aid for Young Scientists (B) (No. 22791319) from the Ministry of Education, Culture, Sports, Science and Technology, Japan. Disclosures: Authors have nothing to disclose with regard to commercial support.

Received for publication March 25, 2013; revisions received July 12, 2013; accepted for publication July 18, 2013; available ahead of print Sept 9, 2013.

Address for reprints: Mitsumasa Kawago, MD, Department of Thoracic and Cardiovascular Surgery, Wakayama Medical University, 811-1 Kimiidera,

Wakayama 641-8509, Japan (E-mail: mkawago@wakayama-med.ac.jp). 0022-5223/\$36.00

Crown Copyright (C) 2014 Published by Elsevier Inc. on behalf of The American Association for Thoracic Surgery

http://dx.doi.org/10.1016/j.jtcvs.2013.07.039
}

reduction surgery has failed to show a survival benefit. ${ }^{3}$ If regeneration of emphysematous lung tissue can be induced, this could provide a promising option for the treatment of pulmonary emphysema.

Regeneration of emphysematous lung tissue has already been reported in animal experiments. ${ }^{4-9}$ In 2005, Morino and colleagues ${ }^{8}$ reported that intra-alveolar administration of gelatin-embedded, sustained-release (SR) basic fibroblast growth factor (bFGF) induced regeneration of alveolar structure in an experimental canine emphysema model. In 2008, Mori and colleagues ${ }^{9}$ also reported pulmonary regeneration after administration of SR-bFGF via the pulmonary artery in a canine emphysema model.

SR-bFGF appears to be a promising treatment for the regeneration of emphysematous lungs. However, intra-airway or intra-arterial administration of the gelatin hydrogel, rather than bFGF solution, may be harmful to patients. The administration of gelatin hydrogel may cause temporary deterioration in pulmonary function because of atelectasis or pulmonary embolism. ${ }^{8,9}$ We believe that this obstacle to the clinical use of SR-bFGF in patients with pulmonary emphysema can be overcome by using an alternative route of administration.

Patients with severe pulmonary emphysema often develop pneumothorax. ${ }^{10}$ Most of these patients have intractable disease because of severe destruction of the 


\section{Abbreviations and Acronyms \\ $\mathrm{bFGF}=$ basic fibroblast growth factor \\ $\mathrm{FGF}=$ fibroblast growth factor \\ $\mathrm{LM}=$ mean linear intercept \\ $\mathrm{SR}=$ sustained release}

lung parenchyma. We previously reported intrapleural administration of large amounts of diluted fibrin glue for the treatment of intractable pneumothorax. ${ }^{11}$ Diluted fibrin glue is easily introduced into the pleural cavity via a drainage tube, and can be distributed throughout the pleural space. This procedure is widely used in patients with high-risk pneumothorax. ${ }^{12,13}$

We planned intrapleural administration of SR-bFGF in patients with pneumothorax who were undergoing intrapleural administration of a large amount of diluted fibrin glue. By adding SR-bFGF to the diluted fibrin glue, SR-bFGF can easily be delivered to the entire pleural surface of the affected lung. We speculate that this route of administration will not harm our patients and may, therefore, open the door to regeneration therapy in patients with pulmonary emphysema. However, there are no reports describing the effectiveness of intrapleural administration of SR-bFGF in terms of regeneration of emphysematous lungs.

In this study, we investigated the effectiveness of intrapleural administration of SR-bFGF in an animal model of elastase-induced pulmonary emphysema. This study preceded our clinical trial of intrapleural administration of SR-bFGF in patients with pulmonary emphysema.

\section{METHODS}

\section{Preparation of the Elastase-Induced Rat Pulmonary Emphysema Model}

The study design was approved by the animal experiment ethical review board of our institute (No. 363). The experiments were performed in accordance with the Guide for the Care and Use of Laboratory Animals. ${ }^{14}$ Eighteen 14-week-old female Sprague-Dawley rats were used. The animals were divided into 4 groups: the completely untreated group (untreated group, $\mathrm{n}=2)$, the elastase-only group $(\mathrm{n}=2)$, the group that received SR-bFGF $\left(\mathrm{FGF}^{+}\right.$group, $\mathrm{n}=7$ ), and the group that received gelatin hydrogel without bFGF ( $\mathrm{FGF}^{-}$group, $\mathrm{n}=7$ ). Animals in the untreated group were used to obtain histologic findings of normal lungs, and were observed for 10 weeks without undergoing any experimental procedures.

Sixteen rats were anesthetized by isoflurane inhalation with spontaneous breathing. The skin over the ventral aspect of the neck was incised, and the trachea was exposed. A 26-gauge needle was used to inject $250 \mathrm{U}$ (total volume, $500 \mu \mathrm{L}$ ) of porcine elastase (Nacalai Tesque, Kyoto, Japan) into the trachea. The neck skin was sutured closed, and the rats were rotated several times to distribute the elastase solution equally to both lungs. The rats were then observed for 8 weeks while they developed pulmonary emphysema. ${ }^{15}$ Development of pulmonary emphysema was confirmed by the preliminary study.

\section{Preparation of SR-bFGF}

Gelatin was isolated from bovine bone collagen by an alkaline process using $\mathrm{CaOH}_{2}$ (Nitta Gelatin Company, Osaka, Japan). Gelatin hydrogel sheets were prepared by glutaraldehyde cross-linking of gelatin, as previously described. ${ }^{16}$ The sheets were crushed into small particles, and particles smaller than $53 \mu \mathrm{m}$ in diameter were collected using a mesh filter. The gelatin hydrogel particles were freeze dried and sterilized using ethylene oxide.

Human recombinant bFGF (Kaken Pharmaceutical Co, Tokyo, Japan) was incorporated into the gelatin hydrogel particles just before the experiment. For each rat, $200 \mu \mathrm{g}$ of bFGF diluted in $20 \mu \mathrm{L}$ of saline was combined with $20 \mathrm{mg}$ of freeze-dried gelatin hydrogel particles, followed by incubation for 1 hour at room temperature. Gelatin hydrogel particles combined with $20 \mu \mathrm{L}$ of saline but no bFGF were also prepared as a control. The gelatin hydrogel particles were mixed with $500 \mu \mathrm{L}$ of fibrin glue solution (Beriplast P Combi-Set; CSL Behring, Tokyo) just before administration.

\section{Intrapleural Administration of SR-bFGF}

At 8 weeks after intra-airway administration of porcine elastase, 14 of the 16 elastase-treated rats were reanesthetized. The other 2 rats were not reanesthetized (the elastase-only group). A small skin incision was made on the left side of the chest, the intercostal muscles were incised, and diluted fibrin glue containing gelatin hydrogel particles was administered via the left pleural cavity. The incisions were closed as quickly as possible, and the rats were manually rotated several times to distribute the gelatin hydrogel particles throughout the pleural cavity. Seven rats received gelatin hydrogel particles with $\mathrm{bFGF}\left(\mathrm{FGF}^{+}\right.$group), and the other seven received gelatin hydrogel particles without $\mathrm{bFGF}\left(\mathrm{FGF}^{-}\right.$group).

\section{Histologic Examination}

All rats were sacrificed at 14 days after the administration of gelatin hydrogel particles, including the rats in the untreated and elastase-only groups. All animals were sacrificed under deep anesthesia using isoflurane. Both lungs and the trachea were excised en bloc. The lungs were immediately fixed in $4 \%$ buffered formalin at a constant airway pressure of $20 \mathrm{~cm}$ $\mathrm{H}_{2} \mathrm{O}$ for 1 week. Both lungs were then cut into sagittal slices and embedded in paraffin. The paraffin blocks were cut into $3-\mu \mathrm{m}$-thick sections and stained with hematoxylin and eosin. The mean linear intercept (LM), which is the standard technique for measuring the size of alveolar spaces, ${ }^{17}$ was used to evaluate the effectiveness of SR-bFGF treatment. The measuring methods for LM were essentially identical as previously reported. In this study, lung slices were observed at $\times 100$ magnification. Photographs of 2776 pixels wide were taken at the upper quarter, center, and lower quarter of the whole lung sagittal section. LMs of 3 horizontal lines were calculated and averaged in each photograph. Then, the median value of LM was adopted in each lung.

\section{Statistical Analysis}

Data are expressed as means and SDs. Differences between groups were analyzed using the unpaired $t$-test. Differences between the right and left lungs were analyzed using the paired $t$-test. $P<.05$ was considered statistically significant.

\section{RESULTS}

A microscopic examination showed severe destruction of the alveolar walls at 10 weeks after intra-airway injection of porcine elastase (Figure 1). The mean LM was approximately twice as long in the elastase-only group as in the untreated group $(116.4 \pm 19.5$ vs $54.5 \pm 3.8 \mu \mathrm{m}$; $P<.0001$ ).

Figure 2 shows the typical microscopic findings of lung tissue after administration of gelatin hydrogel particles. In the $\mathrm{FGF}^{-}$group, the severity of alveolar wall destruction 

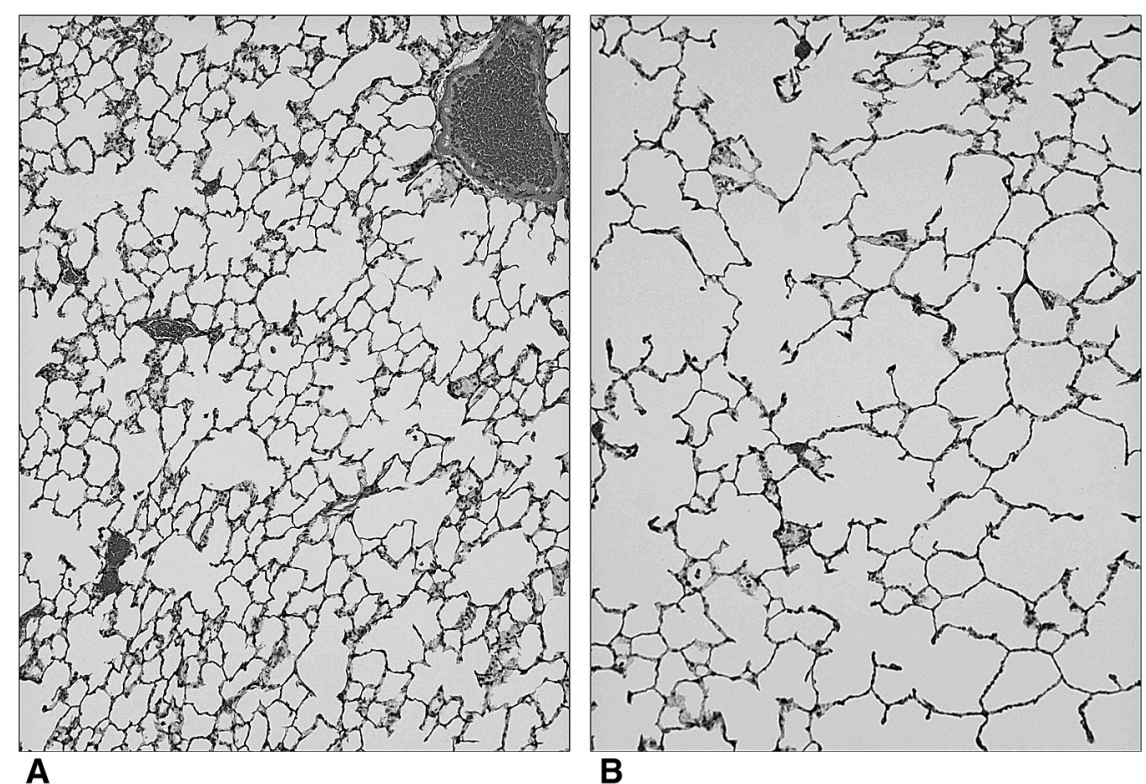

FIGURE 1. Microscopic findings of lung sections. A, Normal lung (untreated group). The mean linear intercept of this section was $57.7 \mu \mathrm{m}$. $\mathrm{B}$, Ten weeks after administration of porcine elastase (elastase-only group). The mean linear intercept of this section was $121.7 \mu \mathrm{m}$. Hematoxylin and eosin staining, $\times 100$.

appears to be identical in the right lung (Figure 2, $A$ ) and the left lung (Figure 2, B). In the $\mathrm{FGF}^{+}$group, alveolar wall destruction appears to be more severe in the right lung (Figure 2, C) than in the left lung (Figure 2, D).

The LMs of the lungs in each group are shown in Figure 3. In the $\mathrm{FGF}^{-}$group, there was no significant difference in mean LM between the right and left lungs
$(119.3 \pm 23.2$ vs $115.0 \pm 14.3 \mu \mathrm{m} ; P=.6237)$. In the $\mathrm{FGF}^{+}$group, the mean LM was significantly longer in the right lung than in the left lung $(114.4 \pm 17.5$ vs $65.1 \pm 7.0 \mu \mathrm{m} ; P=.0005)$. The mean LM of the left lung was also significantly shorter in the $\mathrm{FGF}^{+}$group than in the $\mathrm{FGF}^{-}$group $(P<.0001)$. However, the mean LM was still significantly longer in the left lung of the
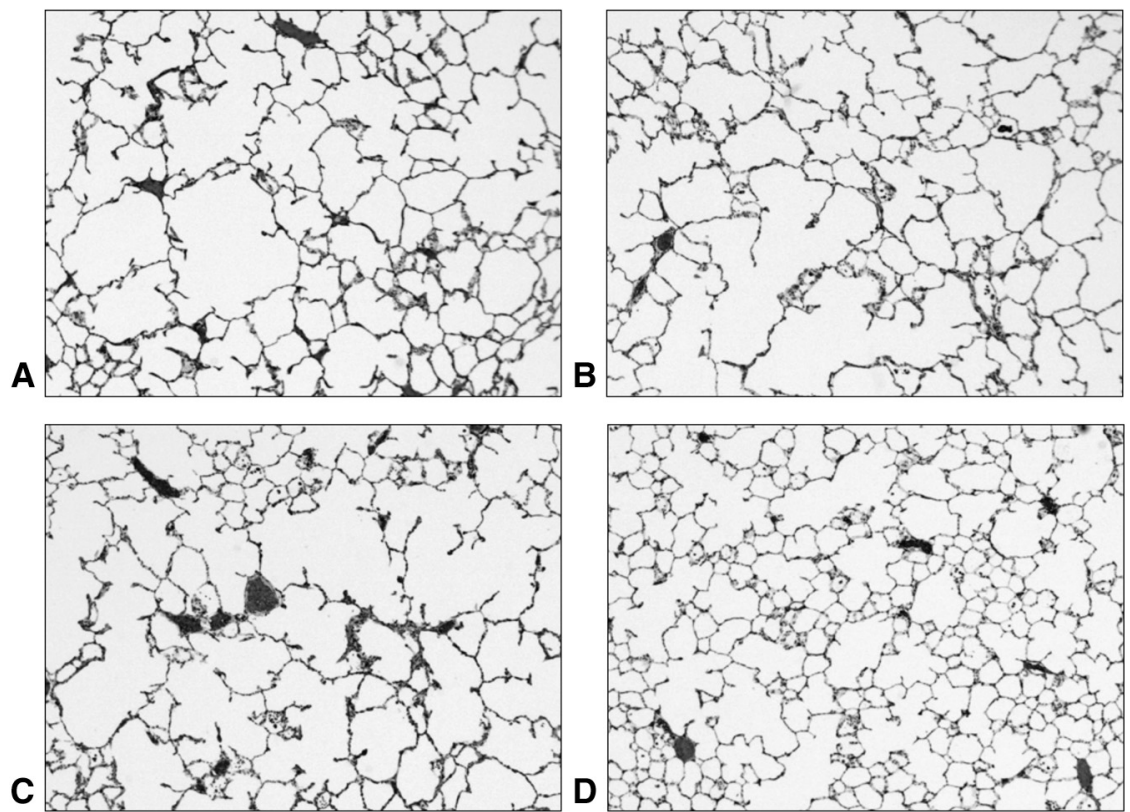

FIGURE 2. Representative microscopic findings of the lung sections of rats who received gelatin hydrogel particles. A, Right lung in the fibroblast growth factor $(F G F)^{-}$group. B, Left lung in the $\mathrm{FGF}^{-}$group. C, Right lung in the $\mathrm{FGF}^{+}$group. D, Left lung in the $\mathrm{FGF}^{+}$group. Hematoxylin and eosin staining, $\times 100$. The mean linear intercepts in these sections were $109.2 \mu \mathrm{m}$ in A, $122.7 \mu \mathrm{m}$ in B, $109.9 \mu \mathrm{m}$ in $\mathrm{C}$, and $67.1 \mu \mathrm{m}$ in $\mathrm{D}$. 


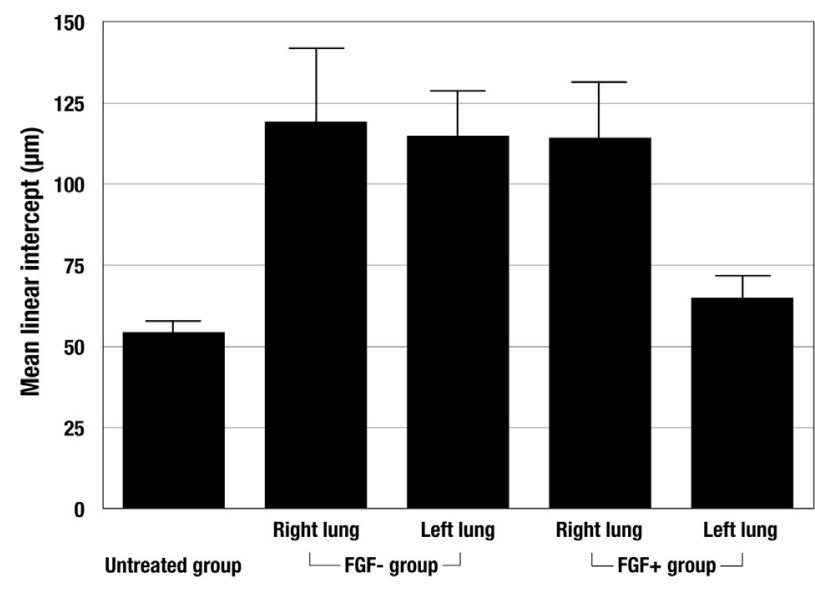

FIGURE 3. Mean linear intercepts of the lung sections of rats that received gelatin hydrogel particles. $b-F G F$, Basic fibroblast growth factor.

$\mathrm{FGF}^{+}$group than in the untreated group $(P=.0027)$. There was no significant difference in the mean LM of the right lung between the $\mathrm{FGF}^{-}$and $\mathrm{FGF}^{+}$groups $(P=.4875)$.

\section{DISCUSSION}

Many researchers have tried to find treatments that can induce regeneration of damaged alveoli. In vivo animal experiments found that administration of stem cells, retinoic acids, hepatocyte growth factor, adrenomedullin, and bFGF has the potential to induce lung regeneration. ${ }^{4-9}$

Only 2 clinical trials have reported on regeneration of damaged alveoli in patients with pulmonary emphysema. In 2012, Stolk and colleagues ${ }^{18}$ reported the results of a randomized controlled trial of the $\gamma$-selective retinoic acid agonist, palovarotene, in 262 patients with pulmonary emphysema. However, they did not find any evidence of lung regeneration in these patients. Weiss and colleagues ${ }^{19}$ reported the results of a randomized controlled trial of allogeneic mesenchymal stem cell administration in patients with pulmonary emphysema. This therapy was reported to be safe, but did not result in lung regeneration. To date, no effective lung regeneration therapy for patients with pulmonary emphysema has been reported.

bFGF is a single-chain polypeptide growth factor that plays a significant role in the process of wound healing. It acts as a strong angiogenic factor that stimulates smooth muscle cell growth, wound healing, and tissue repair. ${ }^{9}$ It is also known to play a role in the development of the fetal lung. ${ }^{20,21}$ bFGF has been used to treat chronic skin wounds, such as pressure ulcers, since 2001, and its effectiveness and safety profile for this use are well known. ${ }^{22,23}$ The biologic half-life of bFGF is less than 50 minutes, ${ }^{24}$ which is too short for a single topical administration to be sufficient, because wound healing processes take longer than a day. ${ }^{25}$ Therefore, administration at least once a day is recommended for the treatment of skin ulcers.
Repeated daily topical administration of bFGF for organ regeneration therapy is almost impossible. Conjugation with a sustained-release material is, therefore, necessary. When SR-bFGF is administered, the bFGF is released slowly as the gelatin hydrogels degrade. The rate of degradation is controlled by the conditions of the crosslink reaction. Our previous experiment found that gelatin hydrogels remained for more than 14 days after administration. ${ }^{16}$ Previous clinical trials reported that SR-bFGF was useful and safe for the treatment of bone fractures, skin defects, and peripheral arterial disease. ${ }^{26-29}$

We believe that SR-bFGF is the most promising potential treatment for inducing lung regeneration in patients with pulmonary emphysema. We speculate that finding a suitable route of administration is the only obstacle to SR-bFGF treatment for pulmonary emphysema. Administration of SR-bFGF via the intrapleural cavity easily overcomes this obstacle. Patients with intractable pneumothorax already have thoracic drains, and administration of SR-bFGF together with intrapleural administration of diluted fibrin glue does not cause any additional harm.

We were unsure whether intrapleural administration of SR-bFGF would result in regeneration of damaged alveoli in emphysematous lungs, as with intra-airway or intra-arterial administration. We, therefore, conducted this animal experiment before starting a clinical trial. There were no unexpected deaths during the experiment, and it appears that intrapleural administration of SR-bFGF is a safe procedure.

Our results show that the mean LM was significantly shorter in the left lung than the right lung in the $\mathrm{FGF}^{+}$ group, indicating that intrapleural administration of SR-bFGF can induce lung regeneration in an elastaseinduced pulmonary emphysema model. The definition of LM indicates that the square of LM is inversely proportional to the number of alveoli. Therefore, our results show that the lungs had 3.2 times as many alveoli after SR-bFGF treatment as before treatment. The mean LM was longer in the left lung of the $\mathrm{FGF}^{+}$group than in the normal control group, indicating that recovery was incomplete. After SR-bFGF administration, the treated lungs had approximately $70 \%$ of the normal number of alveoli. These results were not inferior to those of intra-airway and intra-arterial administration.

The precise mechanism of lung regeneration by SRbFGF is not proved. This study was not designed to prove it. However, Morino et $\mathrm{al}^{8}{ }^{\mathrm{h}}$ have already mentioned the detailed speculation in their report. bFGF is one of the most powerful angiogenic factors and is indispensable for lung development and branching morphogenesis. SR-bFGF might act in the same mechanism as lung development in the fetal stage. ${ }^{21,30}$ They also noticed that bFGF alone can not develop the lung regeneration. They also speculated that the powerful angiogenic effect of 


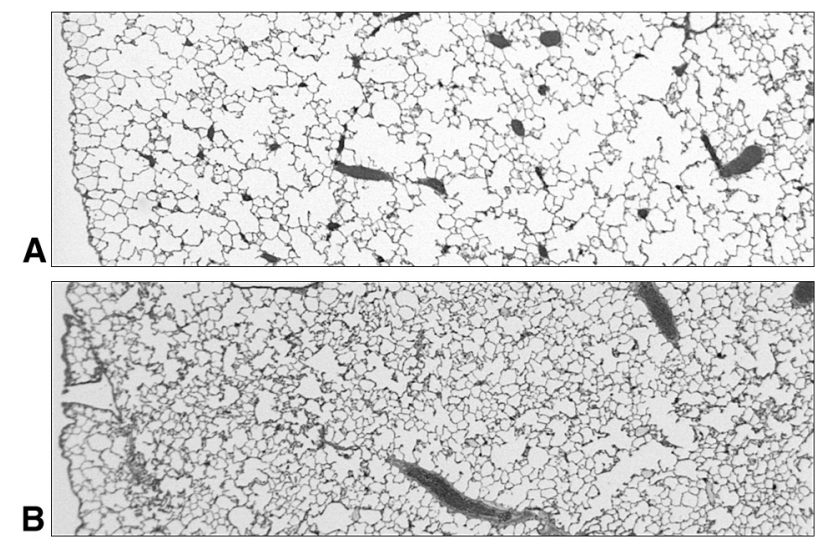

FIGURE 4. Microscopic findings of lung sections of the right lung (A) and left lung (B) in the same rat at low magnification $(\times 20)$. Left side of the photo is the subpleural region and right side is the center of the lung. Lung regeneration appeared to occur in the center of the lung and the subpleural area.

bFGF is also important in the lung regeneration. Increased blood flow caused by SR-bFGF enables to supply sufficient dose of various biological substances that are necessary in the lung regeneration to the parenchyma of the emphysematous lung. There might be another possible explanation in the mechanism of lung regeneration. Pulmonary emphysema is the destruction of the lung parenchyma. bFGF has the strong effect to promote wound healing. Our data might be the result of an accelerated wound healing process.

At the beginning of the study, we were afraid that the regeneration might occur only in a subpleural lesion. Against expectations, the regeneration effects did not look heterogeneous among whole lung (Figure 4). However, rat lung is smaller than human lung. The effect of SR-bFGF might be more limited in the clinical application.

In rats treated with SR-bFGF, the mean LM of the right lung was the same as the mean LM in the $\mathrm{FGF}^{-}$group, indicating that intrapleural administration did not result in recovery of the contralateral lung. However, we believe that the results of this study provided sufficient evidence to start our clinical trial, and we have commenced the "Intrapleural Administration of Sustained-Release Basic Fibroblast Growth Factor in Patients with Chronic Obstructive Pulmonary Disease Accompanied by Pneumothorax: UMIN000004276" trial at our institute.

\section{CONCLUSIONS}

Intrapleural administration of SR-bFGF induced lung regeneration in an elastase-induced pulmonary emphysema model in rats.

We thank Kaken Pharmaceutical Co, Tokyo, Japan, for kindly supplying the basic fibroblast growth factor solution; and CSL Behling, Tokyo, for kindly supplying the fibrin glue.

\section{References}

1. Devereux G. ABC of chronic obstructive pulmonary disease: definition, epidemiology, and risk factors. BMJ. 2006;332:1142-4.

2. Kotloff RM, Thabut G. Lung transplantation. Am J Respir Crit Care Med. 2011; 184:159-71.

3. Fishman A, Martinez F, Naunheim K, Piantadosi S, Wise R, Ries A, et al., National Emphysema Treatment Trial Research Group. A randomized trial comparing lung-volume-reduction surgery with medical therapy for severe emphysema. N Engl J Med. 2003;348:2059-73.

4. Yuhgetsu H, Ohno Y, Funaguchi N, Asai T, Sawada M, Takemura G, et al. Beneficial effects of autologous bone marrow mononuclear cell transplantation against elastase-induced emphysema in rabbits. Exp Lung Res. 2006;32: 413-26.

5. Massaro GD, Massaro D. Retinoic acid treatment abrogates elastase-induced pulmonary emphysema in rats. Nat Med. 1997;3:675-7.

6. Shigemura N, Sawa Y, Mizuno S, Ono M, Ohta M, Nakamura T, et al. Amelioration of pulmonary emphysema by in vivo gene transfection with hepatocyte growth factor in rats. Circulation. 2005;111:1407-14.

7. Murakami S, Nagaya N, Itoh T, Iwase T, Fujisato T, Nishioka K, et al. Adrenomedullin regenerates alveoli and vasculature in elastase-induced pulmonary emphysema in mice. Am J Respir Crit Care Med. 2005;172:581-9.

8. Morino S, Nakamura T, Toba T, Takahashi M, Kushibiki T, Tabata Y, et al Fibroblast growth factor-2 induces recovery of pulmonary blood flow in canine emphysema models. Chest. 2005;128:920-6.

9. Mori N, Gotoh M, Chang SS, Igai H, Misaki N, Yamamoto Y, et al. Reconstruction of emphysematous lung tissue using slowly released basic fibroblast growth factor from gelatin microspheres. ASAIO J. 2008;54:622-6.

10. Melton LJ III, Hepper NG, Offord KP. Influence of height on the risk of spontaneous pneumothorax. Mayo Clin Proc. 1981;56:678-82.

11. Kinoshita T, Miyoshi S, Katoh M, Yoshimasu T, Juri M, Maebeya S, et al. Intrapleural administration of a large amount of diluted fibrin glue for intractable pneumothorax. Chest. 2000;117:790-5.

12. Kinoshita T, Miyoshi S, Suzuma T, Sakurai T, Enomoto K, Yoshimasu T, et al. Intrapleural administration of a large amount of diluted fibrin glue for intractable pneumothorax: a clinical study based on 57 cases: including 2 unsuccessful cases. Jpn J Thorac Cardiovasc Surg. 2003;51:41-7.

13. Light RW. Intrapleural fibrin glue for persistent air leak. In: Pleural Disease. Philadelphia, PA: Lippincott Williams \& Wilkins; 2007:313.

14. Ministry of Health, Labour and Welfare, Japan. Guide for the Care and Use of Laboratory Animals. Available at: http://www.mhlw.go.jp/general/seido/ kousei/i-kenkyu/doubutsu/0606sisin.html [in Japanese].

15. Onclinx C, De Maertelaer V, Gustin P, Gevenois PA. Elastase-induced pulmonary emphysema in rats: comparison of computed density and microscopic morphometry. Radiology. 2006;241:763-70.

16. Tabata Y, Hijikata S, Muniruzzaman M, Ikada Y. Neovascularization effect of biodegradable gelatin microspheres incorporating basic fibroblast growth factor. J Biomater Sci Polym Ed. 1999;10:79-94.

17. Gillooly M, Lamb D, Farrow AS. New automated technique for assessing emphysema on histological sections. J Clin Pathol. 1991;44:1007-11.

18. Stolk J, Stockley RA, Stoel BC, Cooper BG, Piitulainen E, Seersholm N, et al. Randomised controlled trial for emphysema with a selective agonist of the $\gamma$-type retinoic acid receptor. Eur Respir J. 2012;40:306-12.

19. Weiss DJ, Casaburi R, Flannery R, Leroux-Williams M, Tashkin DP. A placebo-controlled randomized trial of mesenchymal stem cells in chronic obstructive pulmonary disease. Chest. 2013;143:1590-8.

20. Gospodarowicz D, Ferrara N, Schweigerer L, Neufeld G. Structural characterization and biological functions of fibroblast growth factor. Endocr Rev. 1987; 8:95-114.

21. Baird A, Ueno N, Esch F, Ling N. Distribution of fibroblast growth factors (FGFs) in tissues and structure-function studies with synthetic fragments of basic FGF. J Cell Physiol Suppl 1987;Suppl 5:101-6.

22. Ishibashi Y, Harada S, Takemura T, Sugawara S, Miyagawa T. Clinical effect of KCB-1 (bFGF) in patients with skin ulcer in the 12 weeks administration. Rinsho-Iyaku. 1996;12:2117-29.

23. Ichioka S, Ohura N, Nakatsuka T. The positive experience of using a growth factor product on deep wounds with exposed bone. J Wound Care. 2005;14: $105-9$.

24. Lazarous DF, Scheinowitz M, Shou M, Hodge E, Rajanayagam S, Hunsberger S, et al. Effects of chronic systemic administration of basic fibroblast growth factor on collateral development in the canine heart. Circulation. 1995;91: $145-53$. 
25. Schaper W, De Brabander M, Lewi P. DNA synthesis and mitoses in coronary collateral vessels of the dog. Circ Res. 1971;28:671-9.

26. Takagi G, Miyamoto M, Tara S, Takagi I, Takano H, Yasutake M, et al. Controlled-release basic fibroblast growth factor for peripheral artery disease: comparison with autologous bone marrow-derived stem cell transfer. Tissue Eng Part A. 2011;17:2787-94.

27. Kusuhara H, Itani Y, Isogai N, Tabata Y. Randomized controlled trial of the application of topical b-FGF-impregnated gelatin microspheres to improve tissue survival in subzone II fingertip amputations. J Hand Surg Eur Vol. 2011;36: 455-60.
28. Kawaguchi H, Oka H, Jingushi S, Izumi T, Fukunaga M, Sato K, et al. A loca application of recombinant human fibroblast growth factor 2 for tibial shaft fractures: a randomized, placebo-controlled trial. J Bone Miner Res. 2010;25: 2735-43.

29. Kawaguchi H, Jingushi S, Izumi T, Fukunaga M, Matsushita T, Nakamura T, et al. Local application of recombinant human fibroblast growth factor-2 on bone repair: a dose-escalation prospective trial on patients with osteotomy. J Orthop Res. 2007;25:480-7.

30. Bikfalvi A, Klein S, Pintucci G, Rifkin DB. Biological roles of fibroblast growth factor-2. Endocr Rev. 1997;18:26-45. 